\title{
El proceso de enseñanza a través de sitios de redes sociales: hacia un nuevo modelo o estilo de enseñanza
}

\author{
Miguel Mateos-Aparicio Palacios \\ Profesor de Educación Secundaria \\ mi_ma_pa@terra.es
}

Recibido: $24 / 07 / 2012$

Aceptado: 23/10/2012

\begin{abstract}
Resumen
La incorporación de las redes sociales al ámbito educativo abre nuevos caminos de investigación, especialmente por las posibilidades de comunicación, interacción y socialización que ofrecen, así como por la multiplicidad de recursos que ponen al servicio de la enseñanza y del aprendizaje, pese a que en esencia son espacios informales cuya dinámica de acción está alejada de los sistemas de control propios de la enseñanza formal y reglada. El presente artículo muestra parte de un trabajo fin de máster sobre el uso educativo de las redes sociales, en concreto se centra en aspectos relativos a la implementación del proceso de enseñanza, como el rol del docente, modelos y/o estilos de enseñanza y los posibles enfoques curriculares que subyacen de su uso educativo. Para ello abordamos el estudio del caso "Econogargallo". Palabras clave: Sitios de redes sociales, redes sociales, estilos de enseñanza, enfoques curriculares.
\end{abstract}

\section{The teaching process trhough social networks: towards a new model or style of teaching}

\begin{abstract}
The use of social networks for the educational field opens new ways of investigation, because of the many possibilities in communication, interaction and socialization that they offer, as well as for the variety of resources and tools for the teaching and learning process, although they are, essentially, informal environments whose dynamics of action is far from the systems of the formal education control. This article shows a part of a final thesis report that focused on the educational use of social networks. In particular, it deals with aspects related to the teaching process, as the teacher's role, the teaching models and possible curriculum approaches that social networks can offer. For this, we study the case "Econogargallo".

Keywords: Social networking sites, social networks, teaching styles, curriculum approaches.

\section{Referencia normalizada}

MATEOS-APARICIO PALACIOS, Miguel (2012): "El proceso de enseñanza a través de sitios de redes sociales: hacia un nuevo modelo o estilo de enseñanza". Estudios sobre el mensaje periodístico. Vol. 18, núm. especial noviembre, págs.: 617-627. Madrid, Servicio de Publicaciones de la Universidad Complutense.
\end{abstract}

Sumario: 1. Introducción; 2. Universo de estudio y metodología; 3. Resultados; 4. Conclusiones; 5 . Referencias bibliográficas.

\section{Introducción}

Resulta innegable que las TIC, con Internet como elemento nuclear, están modificando los esquemas sociales, económicos, políticos, culturales y educativos tradicionales. Su impacto se considera de tal magnitud que se habla de una nueva sociedad, la Sociedad de la Información, Sociedad del Conocimiento o Sociedad Red (Castells, 2000), e incluso de una nueva era: la Era Digital o la Era Internet (Marqués, 2007).

En el ámbito educativo, al margen de la tecnificación de las aulas, la mediación de las TIC ha permitido nuevas modalidades formativas (elearning, blended-learning, 
aprendizaje ubicuo), así como la implementación de nuevos modelos y/o estilos de enseñanza-aprendizaje, intencionadamente alejados de los modelos academicistas, unidireccionales y nemotécnicos. Ejemplos de ello son el aprendizaje digital o interactivo (Silva, 2003: 586), el modelo CAIT -constructivo, auto-regulado, interactivo y tecnológico- (Martín Patino, 2003) o el aprendizaje colaborativo, y, últimamente, con el boom de la Web 2.0, y especialmente de las redes sociales (RRSS), ha vuelto a tomar impulso el concepto de aprendizaje social según la concepción sociocultural de Vigotsky (2000), que entiende el aprendizaje como un fenómeno social en el que la adquisición de nuevo conocimiento es posible gracias a las interacciones con los otros.

El argot cyber ha popularizado el término redes sociales (RRSS) para referirse en realidad a lo que técnicamente son sitios de redes sociales (SRS), entendidos como entornos virtuales que a) permiten la interacción entre distintos usuarios con muy distintos fines, a partir de la cual b) se fomenta la creación de comunidad, y a través de la cual c) se intercambia información. Estos tres factores constituyen indicadores pedagógicos que influyen directamente en la efectividad del aprendizaje, como han defendido Freinet, Freire o el propio Vigotsky, entre otros.

Para posibilitar la interacción, los SRS ofrecen una cada vez más sofisticada infraestructura tecnológica, basada en la llamada "arquitectura de la participación" (O'Reilly, 2005) propia de la Web 2.0, por la que todas las herramientas se ponen al servicio de la colaboración y el intercambio, sin limitaciones para la libre participación' ${ }^{1}$. Así pues, bajo un mismo espacio web encontramos chats, blogs, comentarios, espacios para compartir archivos en diferentes formatos, mensajería interna, lista de contactos, etc., además de un sinfín de aplicaciones. Los SRS se erigen, en este sentido, como espacios integradores de tecnología, aunque, evidentemente, no todos ofrecen las mismas herramientas y, por tanto, tampoco las mismas posibilidades para la interacción.

No obstante, en el ámbito educativo, la Web 2.0 en general, y en particular las RRSS, se han asociado a un modelo de enseñanza más colaborativa y abierta (Marqués, 2010) y a un enfoque crítico del currículum ${ }^{2}$, más democrático y participativo, que pretende un alumno autónomo, activo e interactivo con los recursos didácticos, los espacios de aprendizaje, sus compañeros y los profesores.

Precisamente, y pese a que la literatura tecnodidáctica ha relegado a veces al profesor a un segundo plano, focalizando todo su interés en el aprendizaje y el discente, el rol del profesor resulta fundamental en este modelo de enseñanza, puesto que no solo orienta en el proceso y ofrece fuentes de información, sino que posibilita los recursos; establece los canales de comunicación; prioriza unos elementos del currículum frente a otros, y diseña y rediseña actividades de construcción conjunta de conocimiento, así como sistemas de auto y de co-evaluación. Además, la evaluación considera tan importante el proceso como el resultado. Ahora bien, la toma de decisiones

${ }^{1}$ Hay autores que consideran que la Web 2.0 ha convertido al usuario en el "rey" de internet. Véase Nafría (2007)

2 Sobre los enfoques curriculares pueden consultarse Grundy, S. (1991); Estebaranz (1999); Fueyo Gutiérrez, A. y Lorenzo, Y. (2006), y Fueyo Gutiérrez, A. y Rodríguez Hoyos (2006), entre otros. 
que supone el diseño curricular no es un acto neutro, pues está sujeta, inevitablemente, a condicionantes subjetivos como la concepción que de la enseñanza y del aprendizaje tenga el docente, así como el modelo curricular que pretenda implementar, y ello pese a que en la enseñanza reglada se debe respetar el currículum básico legislado oficialmente por los gobiernos de turno.

Considerando el estado de la cuestión respecto a los SRS y a la enseñanza y al currículum expuesto en los párrafos anteriores, en nuestra investigación nos planteamos tres interrogantes principales: si realmente los SRS son útiles didácticamente, qué modelos o estilos de enseñanza pueden favorecer y de qué manera obligan al docente a redefinir su rol.

\section{Universo de estudio y metodología}

La red social objeto de estudio fue Econogargallo ${ }^{3}$, albergada en Ning, un SRS concebido desde la verticalidad o diversidad social, frente al concepto de uniformidad en que se apoyan los sitios generalistas como Facebook, Twitter o Myspace. Ning forma parte de lo que se ha llamado redes sociales temáticas o verticales, por permitir la creación de redes propias, independientes y cerradas, en función de intereses concretos. Econogargallo fue creada por el Departamento de Economía del I.E.S. Pablo Gargallo de Zaragoza en 2008 y en ella interactúan alumnos de Bachillerato que cursan alguna de las materias asignadas a dicho Departamento. Se trata, por tanto, de una red social vertical y privada, integrada en una enseñanza reglada, formal y presencial.

Durante el curso académico objeto de investigación, el 2009/2010, participaban 120 alumnos y 5 profesores. Todos ellos podían interactuar libremente en los espacios comunes de comunicación, así como en los espacios propios de los grupos a los que pertenecían. En concreto, se habían creado ocho grupos: seis se correspondían con asignaturas y/o cursos específicos; otro grupo había sido destinado a trabajos y proyectos de investigación, y, por último, también se había creado un grupo de ex-alumnos.

Para nuestra investigación tomamos como referencia el modelo pedagógico " $\mathrm{com}$ munity of inquiry" o "comunidad de indagación" de Garrison y Anderson (2005)4, el cual, a partir de procesos formativos mediados por ordenador, analiza las tres dimensiones básicas de todo acto didáctico formal: social, cognitiva y docente, entendidas éstas como presencias textualmente manifestadas, por lo que su estudio lo abordan desde las técnicas del análisis del contenido, a través de un sistema de categorías e indicadores creado para cada dimensión y que aplican al mensaje completo como unidad de análisis, por ser éste "objetivamente identificable" (Rourke et al., 2001).

Al situar nuestra investigación en el análisis del discurso, descartamos los espacios audiovisuales de interacción y nos centramos en los espacios de comunicación asincrónica de base textual, tanto en los abiertos a toda la comunidad de aprendizaje

${ }^{3}$ Referencia web: http://econogargallo.ning.com

4 El modelo de "comunidad de indagación" fue desarrollado en la llamada Escuela de Alberta, donde además de Garrison y Anderson se encontraban otros destacados componentes como Rourke, Archer y Gunawaderna. Para más información puede consultarse http://edutechwiki.unige.ch/en/Community_of_inquiry_model. 
(notas, foro general, blogs y comentarios en los perfiles personales), como en los específicos de los grupos (foros y comentarios). Como unidad básica de análisis escogimos también el mensaje completo, aunque en función de las secuencias de contenido, asignamos diferentes categorías e indicadores a un mismo mensaje.

Respecto a la metodología aplicada, hemos seguido procedimientos propios de la etnografia virtual, aunque hemos combinado técnicas cuantitativas y cualitativas, pues la investigación social es compleja en sí y exige la combinación de ambas técnicas (Callejo y Viedma, 2005:49).

Desde posiciones cuantitativas, hemos partido de un sistema de categorización de análisis del contenido ya pre-establecido, hemos realizado mediciones y ofrecemos, por consiguiente, estadísticas de los niveles de presencia docente en los espacios comunes de comunicación.

Desde posiciones cualitativas, considerando que no existen códigos universales, pues el proceso de codificación textual no solo es subjetivo sino que también depende de las peculiaridades que presente la comunidad objeto de estudio, hemos reconfigurado el modelo de referencia infiriendo categorías e indicadores nuevos a partir de la observación periférica. Ello exigió una metodología inductiva, como también el estudio de las actividades de aprendizaje. Por último, también realizamos una entrevista al administrador de la red con el fin de comprender más detalladamente la organización de la red y los usos didácticos de los diferentes espacios de comunicación.

En la siguiente tabla queda reflejado nuestro sistema de categorías e indicadores para la dimensión docente, el cual puede ser válido para entornos virtuales socializadores que se integran en la dinámica de procesos de enseñanza-aprendizaje formales y presenciales.

\begin{tabular}{|c|c|c|}
\hline CATEGORÍAS & INDICADORES & CÓDIGO \\
\hline $\begin{array}{c}\text { DISEÑO } \\
\text { CURRICULAR }\end{array}$ & $\begin{array}{l}\text { Fijar contenidos y objetivos } \\
\text { Establecer medios didácticos }\end{array}$ & $\begin{array}{l}\text { D-DC-FC } \\
\text { D-DC-ME }\end{array}$ \\
\hline $\begin{array}{l}\text { ORGANIZACIÓN Y } \\
\text { CONTROL }\end{array}$ & $\begin{array}{l}\text { Establecer calendario y temporalización } \\
\text { Establecer normas y velar por ellas } \\
\text { Atender cuestiones técnicos y organizativos } \\
\text { Anunciar cambios y novedades }\end{array}$ & $\begin{array}{l}\text { D-OC-CT } \\
\text { D-OC-EN } \\
\text { D-OC-AT } \\
\text { D-OC-CN }\end{array}$ \\
\hline $\begin{array}{l}\text { ENSEÑANZA } \\
\text { DIRECTA }\end{array}$ & $\begin{array}{l}\text { Proponer las actividades } \\
\text { Orientar en la realización de las actividades } \\
\text { Realizar seguimiento de actividades y procurar su } \\
\text { cumplimiento } \\
\text { Evaluar las actividades y el proceso de aprendizaje }\end{array}$ & $\begin{array}{l}\text { D-ED-PA } \\
\text { D-ED-OA } \\
\text { D-ED-SA } \\
\text { D-ED-EV }\end{array}$ \\
\hline $\begin{array}{l}\text { ESTIMULAR LA } \\
\text { PARTICIPACIÓN }\end{array}$ & $\begin{array}{l}\text { Promover el debate } \\
\text { Reconocer el trabajo de los alumnos }\end{array}$ & $\begin{array}{l}\text { D-PA-PD } \\
\text { D-PA-RT }\end{array}$ \\
\hline $\begin{array}{l}\text { OFRECER } \\
\text { INFORMACIÓN }\end{array}$ & $\begin{array}{l}\text { Ofrecer información relacionada con el currículo } \\
\text { Ofrecer información extra-curricular pero relacionada con la } \\
\text { materia y la dinámica del proceso } \\
\text { Ofrecer información académica y profesional } \\
\text { Ofrecer información sobre la red social }\end{array}$ & $\begin{array}{l}\text { D-IN-CU } \\
\text { D-IN-EX } \\
\text { D-IN-AC } \\
\text { D-IN-RS }\end{array}$ \\
\hline
\end{tabular}

Tabla 1: Categorías e indicadores de presencia docente en Econogargallo 


\section{Los resultados}

En la siguiente tabla describimos brevemente el uso didáctico de los espacios de comunicación de base textual objeto de análisis y aportamos cifras y/o porcentajes de mensajes totales, mensajes exclusivamente docentes y nivel presencial de categorías e indicadores, según los códigos expuestos en la tabla 1.

NOTAS (mensajes totales: 22 / mensajes docentes: 22)

A. Categorías e indicadores docentes (en el 91,3\% de los mensajes docentes):

1. Organización y control (50\%): +D-OC-CT (7 mensajes) +D-OC-CN (4)

2. Ofrecer información (31,9\%): +D-IN-RS (4) +D-IN-EX (3)

3. Enseñanza directa : $(18,1 \%):+D-E D-P A(3)+D-E D-O A(1)$

B. Uso didáctico: Espacio de uso docente para ofrecer información de carácter general, aunque también se observan algunos mensajes de enseñanza directa, como alguna propuesta de actividades. También hay contenido social.

PERFILES (mensajes totales: 186 / mensajes docentes: 125)

A. Categorías e indicadores docentes (en el $59,6 \%$ de los mensajes docentes)

1. Organización y control (72,7\%): +D-OC-AT (45 mensajes) +D-OC-EN (29)

2. Enseñanza directa (32,2\%): +D-ED-SA (21) +D-ED-EV (9) +D-ED-OA (4) + D-ED-PA (4)

3. Estimular la participación (4,2\%): +D-PA-RT (5)

4. Ofrecer información (0,9\%): +D-IN-AC (1)

B. Uso didáctico: Espacio de comunicación interpersonal, donde los docentes se comunican con los alumnos de forma individualizada, atendiendo sobre todo a cuestiones de carácter organizativo y de control y ejerciendo enseñanza directa (orientación y seguimiento de actividades, referencias al proceso de evaluación, etc.). Los docentes también generan considerable presencia social.

FORO GENERAL (mensajes totales: 41 /mensajes docentes: 11, 6 propuestas de debate y 5 comentarios)

A. Categorías e indicadores docentes (en el $26,2 \%$ de los mensajes docentes):

1. Enseñanza directa (75\%): +D-ED-OA (3) +D-ED-PA (3) +D-ED-SA (2) +D-ED-EV (1)

2. Estimular la participación (16,7\%): +D-PA-RT (1) +D-PA-PD (1)

3. Organización y control (8,3\%): +D-OC-EN (1)

B. Uso didáctico: Se utiliza para la libre propuesta de líneas de debate, por lo que además de ampliar el currículum formal, abre la posibilidad de conformar un currículum informal paralelo. Sin embargo, la participación discente es escasa, así como la presencia docente para animar los debates. BLOGS (mensajes totales: 323 / posts y comentarios docentes: 24 y22

A. Categorías e indicadores docentes ( $11 \%$ de los mensajes docentes):

1. Ofrecer información (38,9\%): +D-IN-CU (7) +D-IN-EX (7)

2. Enseñanza directa $(36,1 \%):+D-E D-O A(8)+D-E D-S A(3)+D-E D-P A(2)$

3. Organización y control (13,9\%): +D-OC-AT (2) +D-OC-CT (2) +D-OC-EN (1)

4. Estimular la participación (11,1\%): +D-PA-RT (4)

B. Uso didáctico: Media una actividad obligatoria donde los alumnos comparten noticias económicas para comentarlas posteriormente en clase. Se trata de una actividad mixta por combinar en su desarrollo el espacio virtual y espacios físicos. Ello configura también un currículum informal que se considerará en la evaluación. Escasa presencia docente y discreto porcentaje de reacciones a las noticias presentadas, quizá porque el verdadero debate se genera en el aula.

FOROS DE GRUPOS (mensajes totales: 794)

No hemos codificado los niveles de presencia docente en estos espacios por el elevado número de mensajes, lo cual excedía las dimensiones de nuestro trabajo, aunque se han analizado las actividades de aprendizaje. Además, la presencia docente viene a coincidir con las categorías e indicadores del foro general, aunque con mayor grado presencial, debido a que estos foros se han concebido como espacios formales donde impartir el currículum oficial, para lo cual se proponen actividades generalmente obligatorias y mixtas, es decir, exigen la interacción tanto en el SRS como en los espacios físicos.

Tabla 2: Niveles de presencia docente y usos didácticos de los espacios de base textual en Econogargallo 
Según los datos expuestos, hallamos presencia docente en todos los espacios de comunicación textual, aunque es poco recurrente en aquellos cuyo uso didáctico ha sido transmitido claramente a los alumnos, como en los blogs, de participación obligatoria, y en el foro general, de participación voluntaria. Es más frecuente, en cambio, en los perfiles, como espacios de comunicación interpersonal, y en las notas, como espacio para la comunicación general. Estos dos últimos se utilizan especialmente para la organización y control del acto didáctico. En los foros de los grupos, por el contrario, el docente se manifiesta a través de funciones propias de enseñanza directa.

De las cinco categorías docentes que hemos establecido, es diseño curricular la que apenas muestra presencia discursiva, seguramente porque funciones que son inherentes a esta categoría, como establecer planes de estudios, metodología, etc., se comunican en el aula.

Respecto a las categorías docentes más recurrentes, organización y control y enseñanza directa, la primera tiene como principal objetivo marcar claramente la organización y netiqueta de la red social; la segunda es consustancial a la práctica docente y se manifiesta a través de funciones como diseñar, presentar, orientar y evaluar las actividades.

Tampoco muestra un nivel de presencia significativo en los espacios comunes la categoría motivar para la participación, aunque en los foros de los grupos adquiere mayor relevancia sobre todo para reconocer las contribuciones y el trabajo de los alumnos:

Alicia un buen trabajo de investigación antes de contestar así se hace;

cuando quieres trabajas bien sigue así $i$

Respecto a la categoría ofrecer información, el docente escoge los espacios comunes para proporcionar información de interés general, tanto de carácter curricular como extra-curricular, a través de entradas independientes, bien en el foro general, bien en el blog, bien en las notas:

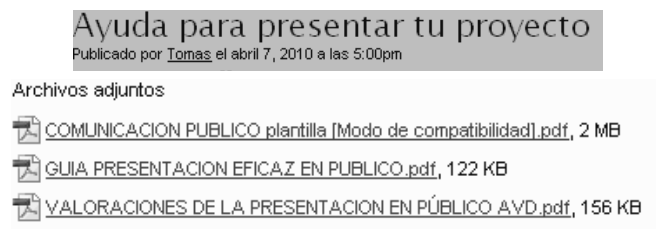

En el blog y en las notas, por ejemplo, hallamos más información extracurricular, aunque relacionada con las actividades que organiza el Departamento de Economía, como excursiones, concursos, etc. Igualmente muchas actividades que se plantean a los grupos van acompañadas de referencias, sobre todo webgráficas:

Web: www.mna.es Ministerio de medio ambiente A través de esta página, accede al programa

En general, de la organización de la enseñanza en la red social y del rol docente manifestado en ella podemos destacar algunas particularidades importantes: 
1. El establecimiento de una netiqueta básica, como identificarse con una fotografía o adscribirse a grupos concretos, para moderar la participación.

2. La interacción entre el SRS y los espacios físicos de enseñanza-aprendizaje obliga al docente a diferenciar claramente entre las acciones formativas que mediará la red social y las que no:

Esta actividad es obligatoria o sustitutiva de otra que os diré en clase.

En esta sección tienes que incluir la siguiente información e incluirla aquí y en papel que se comentará en clase

3. La horizontalidad relacional que permite el SRS respecto a la libre participación de sus integrantes tiene dos consecuencias didácticas: En primer lugar, la participación espontánea introduce un currículum informal más acorde con los intereses de los discentes, como se puede observar en las noticias tratadas en el blog o en los temas propuestos en el foro general; en segundo lugar, esta participación voluntaria se ha de considerar, inevitablemente, en el proceso de evaluación.

4. La sinergia entre contenidos curriculares y contenidos no oficiales, proporcionados estos últimos por los propios alumnos, nos sitúa ante una concepción flexible y abierta del currículum.

5. El constante uso de los medios de comunicación -impresos, digitales- como recursos didácticos que proporcionan información, conceptos, temas de debate y crean opinión crítica, pone en conexión al alumno con la actualidad de su objeto de aprendizaje.

6. La importancia que se concede a las actividades de grupo, entre ellas los proyectos de carácter colaborativo, indica que el docente promueve dinámicas propias del aprendizaje social.

7. Concebir el SRS como espacio común para la interacción de discentes de distintos cursos y niveles académicos, incluidos los ex-alumnos, diluye las fronteras físicas de la organización académica presencial y amplía las posibilidades para generar y compartir conocimiento, lo que podemos asociar también al aprendizaje social.

8. El diseño de actividades mixtas muestra la voluntad de integrar la red social en el diseño global del proceso de aprendizaje.

9. Pedir constantemente opinión personal, debatir los temas en los foros y/o en el aula, proponer temas de repercusión social, etc., reflejan el interés docente por desarrollar sentido crítico ante el propio hecho económico:

¿Porqué crees que los bancos no aydan en la activación de la economía concediendo préstamos?

¿Qué medidas anticrisis implantarías para mejorar nuestro país?

10. El diseño de un sistema de evaluación que recompensa la participación en todos los espacios, ya sea de carácter voluntario u obligatorio, garantiza no discriminar académicamente ninguno de ellos.

11. El desarrollo de un sistema exclusivo de co-evaluación sobre la participación e implicación de los alumnos en la red social muestra, por un lado, el interés docente por implicarles en un proceso conjunto -social- de evaluación, y, por otro, el esfuerzo por transmitir la importancia de las TIC en cuanto espacios y herramientas facilitadores de aprendizaje. Según explicó el docente-administrador en 
la entrevista, cada mes se entrega un "estadillo" con el nombre de los alumnos a cada grupo y éstos deben evaluar las contribuciones de sus compañeros en la red social.

De las consideraciones anteriores, podemos afirmar que en Econogargallo subyace una concepción abierta de enseñanza y un enfoque social e interactivo del currículum -alejados del modelo tradicional transmisor de cultura-, donde se combinan parámetros no directivos, constructivistas y en cierta medida críticos (Estebaranz, 1999):

- No directivos, porque el docente, aunque impone y reitera normas de organización e incluso avisa con sanciones en el caso de no cumplirse, se manifiesta orientando en la experiencia formativa, seleccionando recursos, organizando los espacios de comunicación para la libre participación y reconociendo el trabajo de los alumnos.

- Constructivistas, desde una perspectiva social, porque la dinámica de la red se asienta en la interacción y en la comunicación de las acciones de aprendizaje, tanto individuales como de grupo.

- Críticos, no en el sentido de acercarse al currículum desde posiciones social y económicamente transformadoras, sino en cuanto a despertar interés y espíritu crítico ante un mundo tan complejo como la economía y todas sus repercusiones sociales, entendidas en términos de justicia, bienestar, marginación, etc.

No obstante, debemos considerar algunas apreciaciones a esta concepción abierta de la enseñanza y del currículum.

A. En primer lugar, el currículum oficial está constantemente presente en el discurso docente:

- En la temporalización de actividades:

El plazo es hasta el 20 de mayo......No habeis entregado el trabajo en el tiempo limite

- En las reiteradas referencias al sistema de calificación:

Los primeros tendrán más puntuación. Se valorarán las discusiones y debates que se generen. Lo tendré en cuenta para tu nota en la segunda evaluación

- En la propuesta de actividades marcadas por el propio currículum oficial (proyectos de investigación, de creación empresarial).

B. En segundo lugar, el proceso de evaluación continua primando la calificación de las actividades obligatorias, acorde con el currículum oficial, frente a la participación voluntaria.

C. En tercer lugar, los mecanismos de control siguen en manos del docente: la propuesta de actividades obligatorias, la aceptación o rechazo de los temas para los proyectos, y, en última instancia, la calificación: 
No aceptado.

Ya hay varios sobre la crisis... no te lo aconsejo,

Es muy técnico y te faltan conocimientos para defenderlo

D. En cuarto lugar, los bajos niveles de flujo interactivo de mensajes, no solo en los espacios comunes, sino también en los específicos de algunos grupos, no se corresponden con lo que debiera ser un verdadero proceso social e interactivo de enseñanza-aprendizaje. El docente-administrador aduce que "es dificil debatir temas de economía en profundidad en estas edades y cuando lo hacen es porque se les obliga".

E. En quinto lugar, y según reconoce el administrador de la red, aunque en la programación general del Departamento se contempla oficialmente un apartado de carácter general para la incorporación de las TIC al aula, no se concretan curricularmente ni su aspecto descriptivo (estrategias didácticas, metodología), ni su aspecto conceptual (contenidos que se van a impartir a través de ellas) ni su $a s$ pecto interpretativo (ponderación en el proceso de evaluación). Como afirman Fueyo Rodríguez, Rodríguez Hoyos y Calvo (2011), parece ser habitual que los agentes educativos que ponen en marcha acciones formativas en línea vayan conformando día a día unos procedimientos concretos para afrontar su trabajo, cuyas reglas no se explicitan generalmente, pues constantemente están sometidos a cambios en función de los problemas o demandas que se vayan presentando.

Pese a todo, el hecho de integrar un SRS como espacio de aprendizaje en procesos formales y presenciales es en sí un reto no exento de riesgos, ya que supone un giro de 180 grados en las prácticas docentes si consideramos dos factores importantes:

1) Que el docente comparte las mismas herramientas para la enseñanza que los alumnos utilizan para el aprendizaje.

2) Que el SRS obliga al docente a una constante exposición pública, la cual ha de mantenerse coherente con la identidad que se ha ido forjando en el aula.

Aun consciente de estos riesgos, el docente-administrador de Econogargallo se muestra satisfecho con el proceso y los resultados obtenidos, sobre todo porque " $\mathrm{el}$ alumno está enganchado a la economía, sin enterarse, fuera del aula", que era, en definitiva, su principal objetivo.

\section{Conclusiones}

En primer lugar, Econogargallo muestra cómo los SRS permiten implementar diseños curriculares para procesos formales y presenciales, al concebir cada espacio de comunicación con un uso didáctico concreto y diseñando no solo actividades virtuales sino también mixtas, las cuales favorecen la interacción sinérgica entre los espacios de enseñanza-aprendizaje virtuales y los presenciales.

En segundo lugar, hemos visto cómo la participación espontánea va conformando un currículum informal, más acorde con los intereses de los discentes, que pasa a integrarse con el currículum oficial no solo a través de dinámicas de aprendizaje, sino también mediante su consideración en los procesos de evaluación, lo que nos sitúa 
ante una concepción flexible y abierta del currículum. Por posibilidades como éstas y por el momento- los SRS se alzan como los espacios web que mejor unen aprendizaje formal e informal, aunque en los procesos formales el currículum oficial siga dominando el acto didáctico, como pone de manifiesto un gran porcentaje de las intervenciones docentes.

En tercer lugar, hallamos indicadores de un estilo abierto de enseñanza, como horizontalidad relacional, libertad de acción, flexibilización curricular con aportaciones informales que se considerarán en el proceso de evaluación, etc. No obstante, se echa de menos mayor presencia docente para animar los debates. Convendría explorar estrategias didácticas que motivaran para la interacción y alentaran al intercambio de mensajes, como, por ejemplo, relacionar ideas de diferentes entradas, extraer conclusiones, destacar opiniones diferentes, etc. Los espacios socializadores exigen participación continua y los docentes también deben esforzarse por redefinir su rol en este aspecto.

Por último, añadir que la integración de SRS en procesos formales de enseñanza supone cuanto menos una situación favorable de partida, ya que puede aumentar la motivación y mejorar el ambiente de aprendizaje por ser espacios familiares para los jóvenes y cada vez más para los adultos, aunque, evidentemente, la decisión debe responder necesariamente a criterios didáctico-pedagógicos que persigan un uso cognitivo de estos espacios sociales. Por ello, optar por un SRS (véase Twitter, Facebook, Tuenti, Google+ o Ning, etc., por citar solo algunos de los más populares), supone, en primer lugar plantearse para quién, para qué y cómo lo vamos a utilizar y, en segundo lugar, conocer el medio y sus posibilidades didácticas, o lo que es lo mismo, sus posibilidades para la cognición.

\section{Referencias bibliográficas}

CALLEJO, Javier y VIEDMA, Antonio (2005): Proyectos y estrategias de investigación social: La perspectiva de la intervención. Madrid, McGraw Hill.

CASTELLS, Manuel (2000): La era de la información: Economía, Sociedad y Cultura. Vol. 1 La Sociedad Red. Madrid, Alianza Editorial.

ESTEBARANZ GARCÍA, Araceli (1999): Didáctica e innovación curricular. Universidad de Sevilla.

FUEYO GUTIÉRREZ, Aquilina y LORENZO, Yolanda (2006): Dimensiones pedagógicas y didácticas en el e-learning. En SANTAMERÍA, M. y otros (2006): Principios teóricos y prácticos del e-learning. Madrid, UNED.

FUEYO GUTIÉRREZ, Aquilina y RODRÍGUEZ HOYOS, Carlos (2006): Los procesos de enseñanza-aprendizaje virtuales: hacia una redefinición didáctica de la teleformación. Oviedo, III Simposio Pluridisciplinar sobre Objetos y Diseños de Aprendizaje.

FUEYO GUTIÉRREZ, Aquilina; RODRÍGUEZ HOYOS, Carlos y CALVO, A. (2011): Más allá de la eficacia. investigar la educación en línea desde la etnografia virtual. En APARICI, Roberto (coord.): Conectados en el ciberespacio. Madrid, UNED. 
GARRISON, D. Randy y ANDERSON, Terry (2005): El e-learning en el siglo XXI: Investigación y práctica. Octaedro, Barcelona.

GRUNDY, Shirley (1991): Producto o praxis del currículo. Morata, Madrid.

MARQUÉS, Pere (2007): Usos educativos de Internet (el tercer mundo). DIM (Didáctica y Multimedia), Departamento de Pedagogía Aplicada, Facultad de Educación, UAB.http://dewey.uab.es/PMARQUES/usosred2.htm

MARQUÉS, Pere (2010): La enseñanza. Buenas prácticas. Comunicación. http://www.peremarques.net/actodid.htm DIM (Didáctica y Multimedia), Departamento de Pedagogía Aplicada, Facultad de Educación, UAB.

MARTÍN PATINO, José María y otros (2003): Cómo aprender con Internet. Fundación Encuentro, Madrid. Disponible en red el modelo pedagógico CAIT: http://www.educared.net/InnovacionPedagogica/ModelopedagogicoCAIT.pdf

NAFRÍA, Ismael (2007): Web 2.0, El usuario es el rey. Barcelona: Ediciones Gestión 2000, Planeta de Agostini.

O'REILLY, Tim (2005): Qué es Web 2.0. Patrones del diseño y modelos del negocio para la siguiente generación del software. Boletín de la Sociedad de la Información de Telefónica. http://sociedadinformacion.fundacion.telefonica.com

ROURKE, Liam; ANDERSON, Terry; GARRISON, D. Randy y ARCHER, Walter (2001): Methodological Issues in the Content Analysis of Computer Conference Transcripts. International Journal of Artificial Intelligence in Education, 12(1) 822.

SILVA, Marco (2003): El desafio emergente de la comunicación en la educación presencial y a distancia. En APARICI, Roberto (Coord.): Comunicación educativa en la Sociedad de la Información. Madrid, UNED. pp. 577-596.

VYGOTSKY, Lev Semiónovich (2000): El desarrollo de los procesos psicológicos superiores. Barcelona, Grijalbo.

\section{Miguel MATEOS-APARICIO PALACIOS}

mi_ma_pa@terra.es

Profesor de Educación Secundaria, especialidad en Lengua y Literatura. Máster en Comunicación y Educación en la red. Especialidad: Elearning. UNED. Acreditación como tutor de prácticas del título del máster universitario de profesor de educación secundaria, formación profesional y enseñanzas de idiomas, por la Consejería de Educación de la Junta de Comunidades de Castilla la Mancha. 